\title{
Radioiodoterapia do carcinoma diferenciado da tireoide: impacto radiológico da liberação hospitalar de pacientes com atividades entre 100 e $150 \mathrm{mCi}$ de iodo-131
}

\author{
Radioiodine therapy of differentiated thyroid cancer: radiologic impact \\ of out-patient treatment with 100 to $150 \mathrm{mCi}$ lodine-131 activities
}

Marcelo Tatit Sapienza ${ }^{1,2}$, José Willegaignon ${ }^{1,2}$, Carla Rachel Ono ${ }^{1,2}$, Tomoco Watanabe ${ }^{1,2}$, Maria Inês Calil Cury Guimarães ${ }^{1,2}$, Ricardo Fraga Gutterres ${ }^{3}$, Maria Helena da Hora Maréchal ${ }^{3}$, Carlos Alberto Buchpiguel ${ }^{1,2}$

' Serviço de Medicina Nuclear, Departamento de Radiologia, Faculdade de Medicina, Universidade de São Paulo (FMUSP). ${ }^{2}$ Laboratório de Investigação Médica (LIM/43), Hospital das Clínicas, FMUSP:

São Paulo, SP, Brasil. ${ }^{3}$ Comissão Nacional de Energia Nuclear (CNEN) Rio de Janeiro, RJ, Brasil.
Correspondência para: Marcelo Tatit Sapienza Serviço de Medicina Nuclear, Instituto de Radiologia, HC-FMUSP

Travessa Dr. Ovídio Pires de Campos, s/no - Cerqueira César 05403-010 - São Paulo, SP, Brasil mtsapienza@hotmail.com

Recebido em 6/Nov/2008 Aceito em 5/Fev/2009

\section{RESUMO}

Objetivo: Determinar exposições decorrentes da radioiodoterapia ambulatorial do carcinoma diferenciado da tireoide (CDT) sobre os familiares dos pacientes e o meio ambiente. Métodos: Administraram-se 100 a $150 \mathrm{mCi}$ de $\left({ }^{131}\right)$ Nal para tratamento ambulatorial de 20 pacientes com CDT. Monitorizaram-se com dosímetros termoluminescentes as doses de radiação recebidas por familiares $(n=27)$ e potenciais de dose nas residências. Também foram monitorizadas contaminação de superfície e rejeitos radioativos. Resultados: Registraram-se doses $<1,0$ mSv em 26 acompanhantes e 2,8 mSv em um caso, inferiores ao aceitável para exposições médicas (5,0 mSv/procedimento). Excetuando-se o quarto dos pacientes (média $=0,69 \mathrm{mSv}$ ), determinou-se potencial de dose nas residências $<0,25 \mathrm{mSv}$. A contaminação de superfícies $\left(4,2 \mathrm{~Bq} \cdot \mathrm{cm}^{-2}\right)$ não ultrapassou níveis de liberação, sem representar riscos mesmo em simulações do pior cenário. Os rejeitos radioativos tiveram volume de 2,5 litros e atividade estimada em $90 \mu \mathrm{Ci}$ (média $=4,5 \mu \mathrm{Ci} /$ paciente). Conclusões: Não foi constatado impacto radiológico ao meio ambiente ou aos familiares de pacientes tratados ambulatorialmente com 100 a $150 \mathrm{mCi}$ de iodo-131 e acompanhados por profissionais qualificados. Arq Bras Endocrinol Metab. 2009;53(3):318-25

Descritores

Neoplasias da glândula tireoide; radioisótopos de iodo; radiometria; meio ambiente

\section{ABSTRACT}

Purpose: To evaluate exposure and dosimetry to family members and environment due to outpatient radioiodine therapy of differentiated thyroid carcinoma. Methods: Twenty patients were treated with $100-150 \mathrm{mCi}$ of iodine-131 on an out-patient basis. Family members dosimetry ( $n=27$ ) and potential doses inside the house were measured with thermoluminescent dosimeters. Surface contamination and radioactive wastes were also monitored. Results: Less than $1.0 \mathrm{mSv}$ doses were found in 26 co-habitants and $2.8 \mathrm{mSv}$ in a single case (inferior to the acceptable value of $5.0 \mathrm{mSv} /$ procedure). Potential doses in the houses were inferior to 0.25 $\mathrm{mSv}$, excluding the patients bedroom (mean value $=0.69 \mathrm{mSv}$ ). Surface contamination (mean $=4.2 \mathrm{~Bq} . \mathrm{cm}^{-2}$ ) were below clearance levels. Radioactive wastes generated had a volume of 2.5 liters and a total activity estimated in $90 \mu \mathrm{Ci}$, with a calculated exposure close to the background radiation levels. Conclusions: No radiological impact was detected after iodine therapy with $100-150 \mathrm{mCi}$ on an out-patient basis followed by experienced professionals. Arq Bras Endocrinol Metab. 2009;53(3):318-25.

Keywords

Thyroid neoplasms; iodine radioisotopes; radiometry; environment 


\section{INTRODUÇÃO}

A terapia com iodo-131 é usualmente indicada para complementar o tratamento cirúrgico (tratamento ablativo) ou tratar metástases do carcinoma diferenciado da tireoide (CDT) (1-3). Contudo, ainda há controvérsias sobre as medidas mais apropriadas de radioproteção a serem adotadas com esse tipo de procedimento.

A dificuldade prática de se calcular o potencial de exposição produzido pela incorporação do iodo-131 no organismo do paciente e o desconhecimento da variação deste potencial na vizinhança dos pacientes em virtude da distância e do tempo, bem como a nãoidentificação do público potencialmente exposto a esses pacientes, conduzem à execução de procedimentos de radioproteção por vezes sub ou superestimados. Essas dificuldades, aliadas às diferentes interpretações das recomendações da Agência Internacional de Energia Atômica (IAEA) e da Comissão Internacional de Proteção Radiológica (ICRP) por autoridades normativas de diferentes países, têm levado à confecção de normas e protocolos de tratamento rígidos, imprimindo a obrigatoriedade de hospitalização de todos os pacientes submetidos à terapia com atividades de iodo-131 superiores a $1,1 \mathrm{GBq}[30 \mathrm{mCi}]$ (4-6).

As hospitalizações após a incorporação de altas atividades de iodo-131 muitas vezes não são justificadas por critérios clínicos e não levam em consideração o interesse ou a condição socioeconômica dos pacientes e de seus familiares. Essas práticas levam ao aumento nos custos da terapia e, por vezes, à limitação de acesso aos procedimentos terapêuticos (7).

O levantamento das exposições apresentadas pelos pacientes aos seus familiares durante o tratamento domiciliar do CDT é a forma mais realista de avaliar o impacto radiológico desse procedimento. Neste estudo, faz-se o levantamento dessas exposições para pacientes tratados com atividades entre $100 \mathrm{mCi}$ e $150 \mathrm{mCi}$ de $\left({ }^{131} \mathrm{I}\right) \mathrm{NaI}$ pela primeira vez em nosso país e discute-se, de maneira crítica, as recomendações internacionais e as práticas de radioproteção.

\section{MÉTODOS}

Foram entrevistados 29 pacientes com CDT encaminhados ao Serviço de Medicina Nuclear do Hospital das Clínicas da Faculdade de Medicina da Universidade de São Paulo (FMUSP) para tratamento ablativo complementar após tireoidectomia total. Destes, 20 pacientes foram considerados com condições clínicas e domiciliares adequadas para o tratamento ambulatorial, sendo 17 mulheres e três homens, com idade de 26 a 61 anos $(44 \pm 11$ anos). Atividades de $100 \mathrm{mCi}$ (12 casos) e $150 \mathrm{mCi}$ (8 casos) foram administradas de acordo com a indicação clínica e o perfil de risco do paciente, estabelecidas de maneira independente deste projeto. Após esclarecimentos sobre o teor da pesquisa, todos os pacientes assinaram termo de consentimento livre e esclarecido. O protocolo de pesquisa foi aprovado pela Comissão de Ética em Pesquisa da FMUSP (CAPPesq) e pela Comissão Nacional de Energia Nuclear (CNEN).

As condições domiciliares e o interesse dos pacientes e familiares pelo tratamento em regime ambulatorial, bem como a possibilidade de atenderem aos requisitos de radioproteção e aos cuidados médicos indicados, foram analisados por meio de entrevista conduzida por um médico nuclear e por um supervisor de radioproteção credenciado pela CNEN. Como critérios de inclusão, consideraram-se a presença de suprimento de água e esgoto encanados no domić́lio, a possibilidade de o paciente dormir por cinco dias em quarto separado para permitir seu isolamento em relação às outras pessoas que habitam no mesmo domicílio, a ausência de crianças e mulheres grávidas no domicílio durante o período da terapia, a habilidade de o paciente e seus familiares compreenderem e seguirem as orientações escritas de radioproteção e médicas e a inexistência de comorbidades graves. A dosagem de beta-hCG foi realizada para excluir gestação nas pacientes em idade fértil.

O iodo-131 foi administrado no Serviço de Medicina Nuclear na forma de cápsula por via oral, sob a supervisão de especialista em medicina nuclear e do supervisor de radioproteção, sendo previamente administrado antiemético (metoclopramida) por via intramuscular. A seguir, os pacientes foram orientados a manter jejum por duas horas e liberados para retornar às suas residências em condução própria ou táxi. Prescreveram-se, rotineiramente, antiemético (metoclopramida $10 \mathrm{mg}$ via oral a cada 8 horas) e antiácido (ranitidina $150 \mathrm{mg}$ via oral a cada 12 horas) por três dias após a administração do iodo-131. Uma lista de instruções médicas e de radioproteção, escrita em termos de fácil compreensão, foi entregue aos pacientes, com cópia guardada nos prontuários (Anexo 1). Foi também orientado evitar contatos prolongados com outros indivíduos durante os cinco dias iniciais da terapia. Apesar de não ter sido fixado período máximo de contato, reforçou-se a importância de restringir o período de 
permanência, na distância inferior a 1 metro, ao mínimo tempo necessário para auxiliar nos cuidados do paciente. O médico especialista em medicina nuclear e o supervisor de radioproteção realizaram visitas domiciliares no primeiro e no terceiro dia após a administração do iodo-131, além de contatos telefônicos diários e, quando necessário, por parte dos pacientes.

A dose de radiação recebida por todos os familiares $(\mathrm{n}=27)$ que auxiliaram nos cuidados domiciliares dos pacientes foi monitorada com dosímetros termolumi- nescentes de tórax (TLDs). O potencial de dose dentro do domicílio também foi analisado por dosimetria de área, utilizando TLDs nos locais mais frequentados pelos pacientes (quarto de dormir, sala de estar, cozinha e banheiro). A figura $\mathrm{l}$ apresenta a metodologia de monitoramento dosimétrico utilizada. Para assegurar que as doses medidas fossem representativas das exposições sofridas pelos indivíduos e dos ambientes monitorados, solicitou-se aos pacientes que não alterassem suas rotinas diárias, com exceção dos seguintes cuidados: dormir

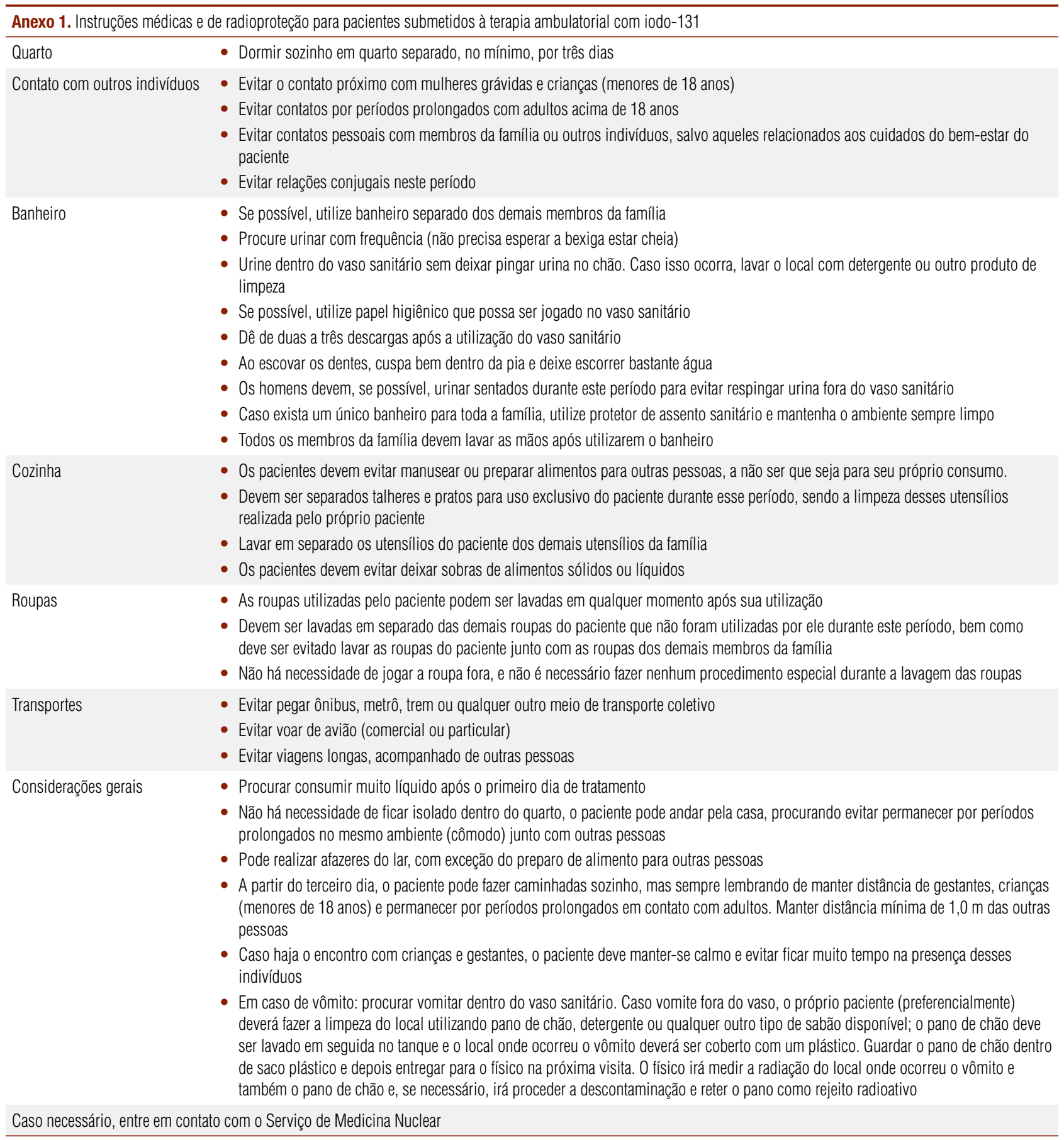


em quartos isolados, evitar manipular alimentos exceto para consumo próprio e evitar permanecer por muito tempo (horas) no mesmo ambiente que seus familiares durante os dois primeiros dias subsequentes à administração da terapia.

O monitoramento das doses foi realizado durante sete dias corridos. A dosimetria pessoal foi iniciada imediatamente após a administração da atividade terapêutica no Serviço de Medicina Nuclear e a de área foi iniciada imediatamente após o retorno do paciente ao seu domicílio. Os TLDs empregados $\left(\mathrm{PRORAD}^{\circledR}\right)$ apresentam resposta linear para doses de $10 \mu \mathrm{Gy}$ a $10 \mathrm{~Gy}$, e a dose efetiva mínima considerada para registro foi de $0,2 \mathrm{mSv}$. As taxas de exposição foram monitoradas a $1,0 \mathrm{~m}$ de distância à frente dos pacientes imediatamente e três dias após a incorporação da atividade de iodo-131.

O detector tipo Geiger-Müller (Victoreen Co., Áustria) com sonda beta-gama (Elimpex-Medizintechnik, Áustria) e sonda de superfície pancake (Elimpex-Medizintechnik, Áustria), calibrado e certificado pelo Instituto de Pesquisas Energéticas e Nucleares (IPEN), foi empregado para monitoramento das taxas de exposição e contaminações superficiais. O nível de contaminação radioativa superficial nos domićlios dos pacientes foi medido com sonda tipo pancake, calculando-se a ati-

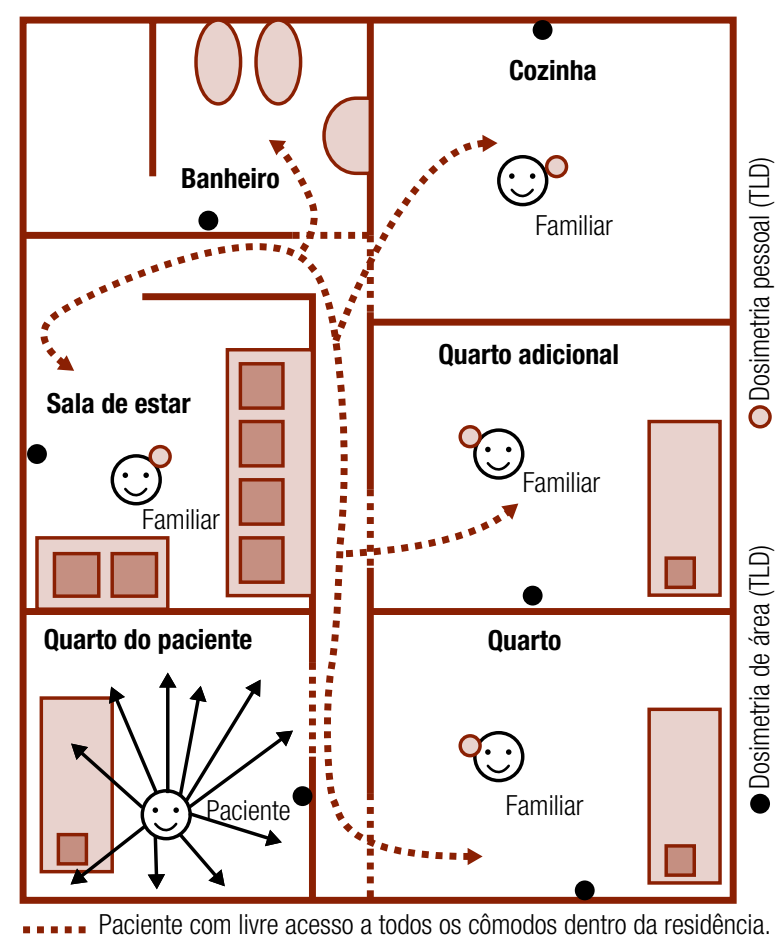

Figura 1. Esquema utilizado para medir a dose de radiação recebida por familiares e o potencial de dose existente dentro das residências. vidade dispersa na superfície dos objetos ou áreas mediante reta de calibração [equação: 1.000 contagens. $\left.\min ^{-1}=0,247 x\left(\mathrm{~Bq} \cdot \mathrm{cm}^{-2}\right)-0,227\right]$. Foram monitorados locais com maior possibilidade de contaminação: cama do paciente, piso, escrivaninha, interruptores, vaso sanitário, talheres, pratos, torneiras e lixeiras.

Todos os resíduos produzidos na residência no período da terapia foram analisados e monitorados, objetivando evitar a liberação de rejeitos com contaminação radioativa superior aos limites indicados nas normas da CNEN (8).

\section{RESULTADOS}

As atividades administradas e as taxas de exposição medidas a 1,0 $\mathrm{m}$ de distância do paciente imediatamente e no terceiro dia após a administração do $\left({ }^{131} \mathrm{I}\right) \mathrm{NaI}$ estão apresentadas na tabela 1 .

Não se observaram reações adversas graves. Notaram-se náusea em nove casos, redução de apetite e astenia em oito casos, dor e edema parotídeo em oito casos

Tabela 1. Principais características clínicas e radiológicas apresentadas pelos pacientes

\begin{tabular}{|c|c|c|c|c|c|}
\hline \multirow[t]{2}{*}{ Paciente } & \multirow{2}{*}{$\begin{array}{l}\text { Sexo } \\
\text { (M/F) }\end{array}$} & \multirow{2}{*}{$\begin{array}{l}\text { Idade } \\
\text { (anos) }\end{array}$} & \multirow{2}{*}{$\begin{array}{l}\text { Atividade } \\
\text { GBq (mCi) }\end{array}$} & \multicolumn{2}{|c|}{$\begin{array}{l}\text { Taxa de exposição } \\
(\mathrm{mR} / \mathrm{h})^{\mathrm{a}}\end{array}$} \\
\hline & & & & Inicial & Final \\
\hline $\mathrm{P1}$ & $\mathrm{F}$ & 59 & $5,55(150)$ & 30,0 & 2,0 \\
\hline P2 & M & 26 & $5,55(150)$ & 30,0 & 2,3 \\
\hline P3 & $\mathrm{F}$ & 38 & $3,70(100)$ & 11,2 & 1,2 \\
\hline P4 & $\mathrm{F}$ & 53 & $3,70(100)$ & 10,9 & $\mathrm{FE}^{\mathrm{b}}$ \\
\hline P5 & $\mathrm{F}$ & 59 & $3,70(100)$ & 15,0 & 4,5 \\
\hline P6 & $\mathrm{F}$ & 36 & $3,70(100)$ & 16,2 & 7,2 \\
\hline P7 & $\mathrm{F}$ & 32 & $3,70(100)$ & 9,8 & 5,6 \\
\hline P8 & $M$ & 54 & $5,55(150)$ & 22,0 & 7,0 \\
\hline P9 & $\mathrm{F}$ & 30 & $3,70(100)$ & 11,0 & 8,0 \\
\hline P10 & $\mathrm{F}$ & 48 & 3,70 (100) & 13,0 & 1,1 \\
\hline P11 & $\mathrm{F}$ & 33 & $3,70(100)$ & FAl & 0,6 \\
\hline P12 & $\mathrm{F}$ & 41 & 3,70 (100) & 10,1 & 4,6 \\
\hline P13 & $\mathrm{F}$ & 44 & 3,70 (100) & 20,0 & 1,5 \\
\hline P14 & $F$ & 33 & 5,55 (150) & 26,5 & 1,4 \\
\hline P15 & $\mathrm{F}$ & 61 & $5,55(150)$ & 23,0 & 2,2 \\
\hline P16 & $\mathrm{F}$ & 54 & 3,70 (100) & $\mathrm{FAl}^{\mathrm{c}}$ & 1,7 \\
\hline P17 & $\mathrm{F}$ & 44 & $5,55(150)$ & 29,0 & 10,0 \\
\hline P18 & $\mathrm{F}$ & 54 & 3,70 (100) & 20,0 & 4,0 \\
\hline P19 & M & 52 & $5,55(150)$ & 26,0 & 4,5 \\
\hline P20 & $\mathrm{F}$ & 32 & $5,55(150)$ & 18,5 & 2,2 \\
\hline
\end{tabular}

${ }^{\mathrm{a}} 1,0 \times 10^{-3} \mathrm{R} \mathrm{h}^{-1}=2,58 \times 10^{-7} \mathrm{C} \mathrm{kg}^{-1} \mathrm{~h}^{-1} ; \mathrm{b} \mathrm{FE}=$ falha do equipamento; ${ }^{\circ} \mathrm{FAl}=$ falha na anotação das informações. 
(com uso de anti-inflamatório não hormonal em um caso), sendo essas reações mais frequentes entre o primeiro e o terceiro dia após tratamento. Houve um episódio de vômito e um paciente com hipersensibilidade à metoclopramida (agitação psicomotora), resolvida com a suspensão da droga.

As doses recebidas pelos familiares foram inferiores a $0,2 \mathrm{mSv}$ em 18 indivíduos, porquanto apenas um paciente apresentou valor máximo de $2,8 \mathrm{mSv}$. As doses registradas em todos os indivíduos expostos são apresentadas na tabela 2 .

Tabela 2. Valores das doses de radiação recebidas pelos familiares dos pacientes durante a terapia domiciliar do CDT

\begin{tabular}{lcc}
\hline Nível de dose (mSv) & $\begin{array}{c}\text { № de indivíduos } \\
\text { expostos }\end{array}$ & Dose média (mSv) \\
\hline$<0,20$ & 18 & $<0,20$ \\
$0,20-0,30$ & 7 & 0,24 \\
$0,31-0,40$ & 0 & 0 \\
$0,41-0,50$ & 0 & 0 \\
$0,51-0,60$ & 1 & 0,60 \\
$\geq 0,61$ & 1 & 2,8 \\
\hline
\end{tabular}

O livre acesso do paciente aos diferentes ambientes dentro da residência leva à existência de potenciais de dose de radiação para outros indivíduos que permaneçam nessas áreas. A dosimetria de área permitiu avaliar esses potenciais, apresentando-se os valores mínimo e máximo e a média das doses encontradas está na tabela 3 .

Tabela 3. Potencial de dose de radiação monitorado nos diferentes ambientes dentro do domicílio dos pacientes

\begin{tabular}{lccc}
\hline \multirow{2}{*}{ Ambiente } & \multicolumn{3}{c}{ Dose (mSv) } \\
\cline { 2 - 4 } & Mínimo & Máximo & Média \\
\hline Quarto do paciente & 0,20 & 1,30 & 0,69 \\
Banheiro & $<0,20$ & 0,60 & $<0,27$ \\
Cozinha & $<0,20$ & 0,30 & $<0,21$ \\
Sala de estar & $<0,20$ & 0,60 & $<0,27$ \\
Quarto do familiar & $<0,20$ & 0,40 & $<0,23$ \\
\hline
\end{tabular}

As contaminações superficiais nas residências variaram entre níveis indetectáveis até valores próximos a $300 \mathrm{~Bq} \cdot \mathrm{cm}^{-2}$, com valor médio de $4,2 \mathrm{~Bq} \cdot \mathrm{cm}^{-2}$ para todas as áreas e objetos monitorados. A tabela 4 apresenta o nível de contaminação nas diferentes localidades monitoradas nas residências de todos os pacientes. As dimensões das áreas contaminadas foram todas limitadas a $100 \mathrm{~cm}^{2}$, sendo consideradas pontos focais.
Os testes de descontaminação provaram que a maioria era removível por métodos simples de limpeza. Independentemente dessa possibilidade, a criação de cenários restritivos (ingestão de objetos contaminados) e cálculos de potenciais de dose indicaram que as contaminações registradas estavam em níveis liberáveis para uso do objeto ou da área contaminada, sem riscos mensuráveis.

Tabela 4. Nível de contaminação superficial encontrado em diferentes ambientes dentro do domicílio dos pacientes

\begin{tabular}{lcccc}
\hline \multirow{2}{*}{ Ambiente } & \multirow{2}{*}{$\begin{array}{c}\text { No de pontos } \\
\text { monitorados }\end{array}$} & \multicolumn{3}{c}{ Nível de contaminação $-\mathbf{B q} \mathbf{~ c m}^{-2}$} \\
\cline { 3 - 5 } & 89 & 0,91 & 4,6 & 1,2 \\
\hline Sala de estar & 126 & 0,91 & 6,6 & 1,3 \\
Cozinha & 100 & 0,91 & 61,2 & 3,4 \\
Quarto do paciente & 147 & 0,91 & 283,9 & 8,9 \\
\hline
\end{tabular}

O volume total de rejeitos radioativos gerado pelos 20 pacientes neste estudo foi de 2,5 litros e a atividade total estimada em $90 \mu \mathrm{Ci}$ de iodo-131. Considerandose o pior cenário (fonte puntiforme) e o potencial de exposição do iodo-131 $\left(0,22 \mathrm{mrem} \cdot \mathrm{mCi}^{-1} \cdot \mathrm{h}^{-1}\right.$ a $\left.1,0 \mathrm{~m}\right)$ (9), estimou-se a taxa de exposição de $0,19 \mu S v \cdot h^{-1}$ a 1 metro e $3,04 \mu S v \cdot h^{-1}$, a $25 \mathrm{~cm}$ da fonte.

A estimativa de dose de radiação recebida por familiares no transporte de pacientes durante o trajeto de retorno do hospital até as residências foi avaliada para dois pacientes, P18 e P19. Com base na taxa de exposição monitorada dentro do veículo, no tempo de transporte e na distância entre o paciente e o familiar (tipicamente $1,0 \mathrm{~m}$ ), a tabela 5 apresenta a estimativa de dose para os casos estudados.

Tabela 5. Dose de radiação recebida por familiares durante 0 transporte de pacientes

\begin{tabular}{lcccc}
\hline Paciente & $\begin{array}{c}\text { Atividade } \\
\text { administrada } \\
\mathbf{G B q}(\mathbf{m C i})\end{array}$ & $\begin{array}{c}\text { Taxa de exposição } \\
\text { dentro do veículo } \\
\left(\mathbf{m R ~ h}^{-1} \mathbf{)}^{\mathbf{a}}\right.\end{array}$ & $\begin{array}{c}\text { Tempo de } \\
\text { transporte } \\
(\mathbf{m i n})\end{array}$ & $\begin{array}{c}\text { Dose } \\
(\boldsymbol{\mu S v})\end{array}$ \\
\hline P18 & $3,7(100)$ & 19 & 45 & 135,4 \\
P19 & $5,55(150)$ & 14 & 55 & 121,9 \\
\hline
\end{tabular}

${ }^{\mathrm{a}} 1,0 \times 10^{-3} \mathrm{R} \mathrm{h}^{-1}=2,58 \times 10^{-7} \mathrm{C} \mathrm{kg}^{-1} \mathrm{~h}^{-1}$.

\section{DISCUSSÃO}

Os pacientes tratados com iodo- 131 podem expor indivíduos que permaneçam no seu campo de radiação ou que sofram incorporação de atividades do radioisótopo excretadas por eles. A hospitalização de pacientes que recebam atividades acima de $30 \mathrm{mCi}$ é estabelecida como medida de radioproteção pelo órgão de re- 
gulamentação nacional (CNEN), visando proteger os familiares, os indivíduos do público e o meio ambiente contra possíveis exposições ou contaminações radioativas. Porém, a internação implica maior custo e pode retardar ou reduzir o número de pacientes tratados. Particularmente em locais que não dispõem de unidades de internação estruturadas para esse tipo de tratamento, o paciente é obrigado a procurar assistência em outras cidades ou mesmo estados, acarretando custos adicionais para o sistema de saúde do país. O presente estudo mostrou que 20 dos 29 pacientes entrevistados apresentavam condições domiciliares adequadas ao tratamento domiciliar, incluindo a presença de esgoto, quarto privativo, ausência de crianças e gestantes. Essa proporção observada em pacientes atendidos pela rede pública de saúde talvez sofra variação em grupos com outras características socioeconômicas. De forma geral, as condições domiciliares no Brasil apresentaram importante modificação nas últimas décadas, havendo de l a 4 habitantes em $71 \%$ dos domicílios, e seis ou mais cômodos em 46\% dos domicílios (10).

Não há consenso sobre as medidas de radioproteção necessárias para conduzir as terapias de modo seguro. As recomendações internacionais da IAEA e da ICRP não determinam hospitalização obrigatória quando empregadas atividades superiores a $30 \mathrm{mCi}$, apontando para critérios de liberação ou internação que incluem as atividades administradas, os potenciais de dose, a condição socioeconômica dos pacientes, o interesse de pacientes e familiares pela internação e a realidade do sistema de saúde local (4,11-14). Desde 1997, os Estados Unidos consideram as condições sociais dos pacientes e o potencial de exposição de seus familiares como critérios para as internações $(9)$.

O presente trabalho teve por objetivo avaliar a viabilidade da terapia do CDT em regime ambulatorial com atividades entre 100 e $150 \mathrm{mCi}$, levando-se em consideração as recomendações internacionais sobre os critérios para liberação hospitalar, bem como o impacto radiológico dessa liberação sobre os familiares dos pacientes e o meio ambiente. O impacto radiológico foi analisado pela mensuração das doses reais recebidas pelos familiares, pelo potencial de dose existente dentro do domicílio, identificação, quantificação e análise dos rejeitos radioativos gerados e presença de contaminação radioativa nos domicílios.

No presente estudo, 26 dos 27 familiares receberam doses inferiores a 1,0 $\mathrm{mSv}$ durante os sete dias do período de monitoramento. A dose de $2,8 \mathrm{mSv}$ foi obser- vada em indivíduo que não seguiu adequadamente as orientações de radioproteção e permaneceu próximo ao paciente por período prolongado, conforme constatado em análise posterior. Em todos os indivíduos monitorados, as doses registradas foram inferiores a $5,0 \mathrm{mSv}$, valor considerado aceitável para familiares que auxiliam nos cuidados dos pacientes (11-15).

$\mathrm{O}$ uso inadequado dos TLDs poderia levar a sub ou superestimativa das doses dos familiares, o que parece improvável diante da relativa homogeneidade dos dados encontrados nos 20 casos. As doses recebidas pelos familiares neste estudo também são similares às encontradas por outros autores em estudos envolvendo terapias do CDT ou hipertireoidismo (16-20). É interessante notar que a terapia do hipertireoidismo é feita ambulatorialmente há mais de 60 anos, com poucas restrições em decorrência das exposições dos familiares. A semelhança no nível de exposição, após terapia do hipertireoidismo e do CDT, ocorre por causa da maior captação e da eliminação orgânica mais lenta do iodo131 nos pacientes com hipertireoidismo.

O período de monitoramento das doses (sete dias consecutivos) é suficiente para estimar cerca de $95 \%$ do total de dose fornecida pelos pacientes, uma vez que estudos anteriores mostraram que em cerca de cinco dias quase toda a atividade administrada aos pacientes é eliminada (7).

Neste estudo não se avaliou a incorporação de atividades de iodo-131 pelos familiares, mas alguns estudos demonstram que as doses provenientes dessas incorporações são muito inferiores às doses provenientes das exposições externas (20-23).

Em relação ao potencial de dose dentro do domicílio, a literatura (19) mostra valores semelhantes aos indicados neste estudo. Com exceção do quarto do paciente, os demais ambientes apresentaram potenciais de dose inferiores a $1,0 \mathrm{mSv}$ durante todo o período de monitoramento. O potencial de dose mais elevado no quarto do paciente $(0,69 \mathrm{mSv})$ é esperado, por ser o local em que o paciente permanece por mais tempo. Da mesma forma, o maior tempo de permanência e a excreção do iodo-131 explicam que as maiores contaminações superficiais sejam encontradas no banheiro e no quarto de dormir.

Independentemente da possibilidade de descontaminação de objetos e áreas, os níveis de contaminação registrados neste estudo estão dentro dos níveis liberáveis, sem necessidade de nenhuma espécie de controle dessas fontes, de acordo com os níveis definidos pela CNEN, 
IAEA ou ICRP de liberação para o uso restrito ou irrestrito de objetos ou áreas contaminadas, em face de cenários e potenciais de dose envolvidos $\left(10 \mu \mathrm{Sv}_{\mathrm{a}} \mathrm{a}^{-1}\right.$ : isenção de controle da fonte, $300 \mu \mathrm{Sv}_{\mathrm{a}} \mathrm{a}^{-1}$ : liberação para uso irrestrito, 1,0 mSv.a ${ }^{-1}$ : liberação para uso restrito) (23-27). Essa análise foi realizada levando-se em consideração o pior cenário, a ingestão de fontes com atividades semelhantes às encontradas nas contaminações e os fatores de dose por unidade de atividade ingerida $\left(\mu \mathrm{Sv} \cdot \mathrm{Bq}^{-1}\right)$.

Outro possível impacto da liberação hospitalar é a possibilidade de os pacientes gerarem rejeitos radioativos que possam expor externa ou internamente qualquer indivíduo que entre em contato com eles. Somente quatro dos 20 pacientes geraram resíduos identificados como rejeitos radioativos, com volume de $2,5 \mathrm{~L}$ e atividade de iodo- 131 estimada em $90 \mu \mathrm{Ci}$. Os rejeitos foram compostos, principalmente, por resíduos de banheiro e de cozinha, gerados pela impossibilidade ou dificuldade de os pacientes atenderem alguns dos cuidados de radioproteção indicados. Desconsiderando a hipótese da ingestão das excretas ou rejeitos, cenário por demais restritivo, o potencial de dose calculado a 1,0 $\mathrm{m}$ de distância dos rejeitos $\left(0,19 \mu \mathrm{Sv} \cdot \mathrm{h}^{-1}\right)$ é comparável à média da taxa de dose recebida anualmente no mundo $\left(2,4 \mathrm{mSv}\right.$ ou $\left.0,27 \mu \mathrm{Sv}_{\mathrm{h}} \mathrm{h}^{-1}\right)(28)$. Mesmo para distâncias menores que $1,0 \mathrm{~m}$, quando considerados $\mathrm{O}$ tempo de manipulação ou o contato com esses rejeitos, o potencial de dose está na ordem de grandeza da radiação natural. $\mathrm{O}$ valor médio de atividade liberada em resíduos domiciliares por paciente $(4,5 \mu \mathrm{Ci})$ é cerca de seis vezes menor que o nível de isenção de controle de fontes radioativas indicado pela IAEA (12). A aderência aos cuidados de radioproteção permite evitar ou minimizar a produção de rejeitos, não se comprovando impacto radiológico mensurável para o meio ambiente ou para indivíduos do público pela liberação de rejeitos para o lixo comum urbano (29-32).

Diversos estudos mostram que o transporte de pacientes do hospital até suas residências é seguro e não oferece exposições além dos patamares aceitáveis quando adotados procedimentos simples de radioproteção $(20,22,33,34)$. A taxa de dose encontrada nesses trabalhos é próxima à estimada no presente estudo, da ordem de $0,10 \mathrm{mSv} \cdot \mathrm{h}^{-1}$.

Como consideração final, cabe ressaltar que as instituições oficiais envolvidas na proteção radiológica assumem não haver comprovação de carcinogênese ou de qualquer outro efeito nocivo à saúde proveniente da irradiação com doses inferiores a $100 \mathrm{mSv}$ (35-40).

\section{CONSIDERAÇÕES FINAIS}

Este estudo demonstrou que, havendo acompanhamento por profissionais habilitados e estrutura de radioproteção adaptada, o tratamento do CDT com atividades entre $100 \mathrm{mCi}$ e $150 \mathrm{mCi}$ de $\left({ }^{131} \mathrm{I}\right) \mathrm{NaI}$ pode ser executado em regime ambulatorial de maneira segura quanto aos aspectos de radioproteção, não havendo impacto radiológico mensurável, tanto ao meio ambiente quanto a qualquer indivíduo isoladamente.

A possível normatização do tratamento em regime ambulatorial pelos órgãos competentes poderia tornar a radioiodoterapia acessível a maior número de pacientes, reduzindo custos tangíveis e intangíveis e podendo inserir fatores positivos para o prognóstico e a qualidade de vida dos pacientes e de seus familiares.

Agradecimentos: os autores deste trabalho agradecem o financiamento concedido pela Fundação de Amparo à Pesquisa do Estado de São Paulo (Fapesp) (Projeto no 06/61556-2) e a autorização para a terapia domiciliar em caráter de estudo concedidos pela CNEN (Ofício no $1083-\mathrm{CGMI} / \mathrm{CNEN}$ ).

Declaração: os autores declaram não haver conflitos de interesse científico neste artigo.

\section{REFERÊNCIAS}

1. Harbert JC, Eckelman WC, Neumann RD. Nuclear medicine: diagnosis and therapy. NewYork:Thieme Medical; 1996.

2. Mazzaferri EL, Kloos RT. Clinical review 128: current approaches to primary therapy for papillary and follicular thyroid cancer. $J$ Clin Endocrinol Metab. 2000;86:1447-63.

3. Maia AL, Ward LS, Carvalho GA, Maciel RMB, Maciel LMZ, Rosário PW, et al. [Nódulos de tireóide e câncer diferenciado de tireóide: consenso brasileiro]. Arq Bras Endocrinol Metab. 2007;51:867-93.

4. International Commission on Radiological Protection. Release of patients after therapy with unsealed radionuclides. ICRP Publication 94. Oxford: Pergamon Press; 2004.

5. Lubin E. Definitive improvement in the approach to the treated patient as a radioactive source. J Nucl Med. 2002;43:364-5.

6. de Klerk JMH. 131I therapy: inpatient or outpatient? J Nucl Med. 2000;41:1876-8.

7. Willegaignon J, Stabin MG, Guimarães MIC, Malvestiti LF, Sapienza MT, Marone M, et al. Evaluation of the potential absorbed doses from patients based on whole-body 131/ clearance in thyroid cancer therapy. Health Phys. 2006;91:123-7.

8. Comissão Nacional de Energia Nuclear. Gerência de Rejeitos Radioativos em Instalações Radiativas. CNEN-NE-6.05, Rio de Janeiro; 1985.

9. Siegel JA, Kroll S, Regan D, Kaminski MS, Wahl LR. A practical methodology for patient release after tositumomab and (131)Itositumomab therapy. J Nucl Med. 2002;43:354-63.

10. Alves JED. As características dos domicílios brasileiros entre 1960 e 2000. Textos para discussão [monografia]. Escola Nacional de Ciências Estatísticas, Ministério do Planejamento, Orçamento e Gestão, Instituto Brasileiro de Geografia e Estatística (IBGE), Escola Nacional de Ciências Estatísticas; 2004. 
11. International Atomic Energy Agency. Radiological protection for medical exposure to ionizing radiation. Safety Guide n. RS-G-1.5. IAEA, Vienna; 2002.

12. International Atomic Energy Agency. International basic safety standards for protection against ionizing radiation and for the safety of radiation sources. Safety Series n. 115 (BSS-115). IAEA, Vienna; 1996.

13. International Commission on Radiological Protection. 1990 Recommendations of the International Comission on Radiological Protection. ICRP Publication 60. Oxford: Pergamon Press; 1990.

14. International Commission on Radiological Protection. Radiological protection and safety in medicine. ICRP Publication 73. Oxford: Pergamon Press; 1996.

15. Comissão Nacional de Energia Nuclear. Diretrizes básicas de proteção radiológica. CNEN-NN-3.01, Rio de Janeiro; 2006.

16. Reiners C, Labmann M. Radioiodine (1311) treatment of hyperthyroidism: radiation protection and quality assurance. Eur $\mathrm{J}$ Nucl Med. 1999;26:683-5.

17. Rutar FJ, Augustine SC, Colcher D, Siegel JA, Jacobson DA, Tempero MA, et al. Outpatient treatment with 131I-anti-B1 antibody: radiation exposure to family members. J Nucl Med. 2001;42:907-15.

18. Cappelen T, Unhjem JF, Amundsen AL, Kravdal G, Folling I. Radiation exposure to family members of patients with thyrotoxicosis treated with iodine-131. Eur J Nucl Med Mol Imaging. 2006;33:81-6.

19. Grigsby PW, Siegel BA, Baker S, Eichling J. Radiation exposure from outpatient radioactive iodine (1311) therapy for thyroid carcinoma. JAMA. 2000;283:2272-4.

20. Pant GC, Sharma SK, Bal CS, Kumar R, Rath GK. Radiation dose to family members of hyperthyroidism and thyroid cancer patients treated with 131I. Radiat Prot Dosim. 2006;118:22-7.

21. Pant GS. Dosimetric and radiation safety considerations in radioiodine therapy. Indian Journal of Nuclear Medicine. 2005;20:1-3.

22. Matheoud R, Reschini E, Canzi C, Voltini F, Gerundini P. Potential third-party radiation exposure from outpatients treated with 131 I for hyperthyroidism. Med Phys. 2004;31:3194-200.

23. Ibis $\mathrm{E}$, Wilson CR, Collier BD, Akansel G, Isitman AT, Yoss RG. Iodine-131 contamination from thyroid cancer patient. J Nucl Med. 1992;33:2110-5.

24. International Atomic Energy Agency. Application of the concepts of exclusion, exemption and clearance. Safety Guide n. RS-G-1.7, IAEA, Vienna; 2004.

25. International Atomic Energy Agency. Release of sites from regulatory control on termination of practices. Safety Guide n. WS-G5.1. IAEA, Vienna; 2006.
26. International Atomic Energy Agency. Clearance levels for radionuclides in solid materials: application exemption principles. IAEA-TECDOC-855, Vienna; 1996.

27. International Atomic Energy Agency. Radiological aspects of non-fixed contamination of packages and conveyances. IAEATECDOC-1449, Vienna; 2005.

28. United Nations Scientific Committee on the Effects of Atomic Radiation. Sources and effects of ionizing radiation. UNSCEAR 2000 Report v. I, Vienna.

29. Prichard HM, Gesell TF, Davis E. lodine-131 levels in sludge and treated municipal wastewaters near a large medical complex. Am J Public Health. 1981;71:47-52.

30. Larsen IL, Stetar EA, Giles BG, Garrison B. Concentration of iodine 131 released from a hospital into a municipal sewer. RSO Magazine. 2001;6:13-8.

31. Fenner FD, Martin JE. Behavior of Na131I and meta(131I) iodobenzylguanidine (MIBG) in municipal sewerage. Health Phys. 1997;73:333-9.

32. Washington State Department of Health. The Presence of radionuclides in sewage sludge and their effect on human health. Environment Radiological Program (WDOH/320-013). Washington, 1997 [acesso 2008 Aug 13]. Disponível em: http://www.doh. wa.gov/ehp/rp/environmental.

33. Marriot CJ, Webber CE, Gulenchyn KY. Radiation exposure for 'caregivers' during high-dose outpatient radioiodine therapy. Radiat Prot Dosimetry. 2007;123:62-7.

34. Moutforda PJ, O'Doherty MJ. Exposure of critical groups to nuclear medicine patients. Appl Radiat Isot. 1999;50:89-111.

35. Aurengo A. Chernobyl: the effects on public health? Physics and Society 2002;31(4). [serial on the Internet]. 2002 [acesso 2008 Aug 13]. Disponivel em: http://units.aps.org/units/fps/newsletters/2002/ october/index.cfm.

36. González AJ. Regulation of low-level radiation. C R Acad Sci III. 1999;322:241-3.

37. Health Physics Society. Radiation risk in perspective. Position Statement of the Health Physics Society, August 2004 [acesso 2008 Aug 13]. Disponível em: http://www.hps.org/documents/ radiationrisk.pdf.

38. American Nuclear Society. Health effects of low-level radiation. Position Statement 41, June 2001. [acesso 2008 Aug 13]. Disponível em: http://www.ans.org/pi/ps/docs/ps41.pdf.

39. US Nuclear Regulatory Commission. Instruction concerning risks from occupational radiation exposure. USNRC. Regulatory Guide 8.29; 1996.

40. RockwellT.The realism project: it's time to get real. Nuclear News. 2004;47:10-2. 